\title{
Estrogen Receptor Genotypes, Menopausal Status, and the Effects of Tamoxifen on Lipid Levels: Revised and Updated Results
}

\author{
DF Hayes ${ }^{1}$, TC Skaar ${ }^{2}$, JM Rae $^{1}, \mathrm{NL} \mathrm{Henry}^{1}, \mathrm{AT} \mathrm{Nguyen}^{2}, \mathrm{~V} \mathrm{Stearns}^{3}, \mathrm{~L} \mathrm{Li}^{2}, \mathrm{~S} \mathrm{Philips}^{2}, \mathrm{Z} \mathrm{Desta}^{2}$ \\ and DA Flockhart ${ }^{2}$; Consortium on Breast Cancer Pharmacogenomics (COBRA)
}

We previously reported that the ESR $1 \mathrm{XbaI}$ genotypes were associated with baseline and tamoxifen-induced serum lipid profiles. The analysis in that study was carried out by PCR followed by restriction-enzyme digestion. After reanalysis using more robust TaqMan assays, the findings related to $\sim 10 \%$ of the genotypes for the ESR $1 \mathrm{XbaI}$ singlenucleotide polymorphism (SNP) were revised. For the other genotypes (i.e., ESR1 PvuII, ESR2, and CYP2D6), the results were nearly identical to those in the previous study. Upon reanalysis, previously reported associations between the ESR1 Xba1 genotypes and baseline triglyceride and lowdensity lipoprotein (LDL) cholesterol levels were no longer observed. Previously reported associations between the ESR1 $\mathrm{XbaI}$ genotypes and tamoxifen-induced changes in levels of total cholesterol, triglycerides, and high-density lipoprotein (HDL) cholesterol were also no longer observed. However, the following observations from the original report did not change: (i) the levels of circulating lipids are lower in women taking tamoxifen; (ii) there is an association between the ESR2-02 genotypes and changes in triglyceride levels; and (iii) neither ESR1 PvuII nor CYP2D6 is associated with any changes in serum lipid concentrations in patients receiving treatment with tamoxifen.

Tamoxifen is a selective estrogen receptor modulator that has tissue-specific estrogen agonistic or antagonistic properties. ${ }^{1}$ We and others have previously reported that tamoxifen induces favorable lipid profiles, presumably because of an estrogenic effect in the liver, mediated through the estrogen receptors $\alpha$ and $\beta(E R \alpha, \beta){ }^{2}$ The genes that encode these receptors (ESR1 and 2 ) are highly polymorphic. ${ }^{3}$ Tamoxifen is a relatively inactive prodrug, but it is converted to active metabolites, including 4-hydroxy tamoxifen and 4-hydroxy-N-desmethyl tamoxifen (designated endoxifen). ${ }^{4}$ Conversion of tamoxifen to endoxifen is highly dependent on the activity of the enzymatic product of CYP2D6, which is also highly polymorphic. ${ }^{5}$ Therefore, we hypothesized that the estrogenic effects of tamoxifen on lipid profile might be associated with inherited single-nucleotide polymorphisms (SNPs) in the genes that encode for the targets or the metabolic enzymes that mediate its activity.

We previously reported ${ }^{2}$ that tamoxifen significantly lowered levels of low-density lipoprotein $(\mathrm{LDL})$ cholesterol $(P<0.0001)$ and increased levels of triglycerides $(P=0.006)$. In postmenopausal women, the ESR1 XbaI GG genotype was associated with relatively greater tamoxifen-induced reductions in total cholesterol level as compared with the GA and AA genotypes $(P=0.03)$. The ESR1 XbaI refers to the SNP rs no. 9340799, and the ESR1 PvuII refers to the SNP rs no. 2234693. In premenopausal women, the ESR1 XbaI GG genotype was associated with a large tamoxifen-induced elevation in triglyceride concentration, whereas tamoxifen-treated women with the AA and AG genotypes had little or no change in triglyceride level $(P=0.002$; gene-dose effect). In contrast, premenopausal women with the ESR1 XbaI AA genotype showed elevated levels of high-density lipoprotein (HDL) cholesterol after 4 months of treatment with tamoxifen, whereas those with the AG genotype showed no change in these levels, and those with the GG genotype showed a reduction ( $P=0.004$; gene-dose effect). In postmenopausal women, the ESR2-02 GG genotype was significantly associated with tamoxifen-induced elevations in serum triglyceride level, whereas there was no discernible change in women with the AG genotype and a reduction in triglyceride level in those with the AA genotype ( $P=0.01$; gene-dose effect). We concluded that estrogen receptor genotyping might be useful in predicting which patients would benefit more from tamoxifen. 
The genotyping data for ESR1 on which our prior report was based were generated by PCR followed by restriction-enzyme digestion (restriction fragment length polymorphism, RFLP), methods previously described by other investigators. ${ }^{6}$ We subsequently redetermined the genotypes using TaqMan assays and found that $\sim 10 \%$ of the genotypes for the ESR1 XbaI SNP, as determined by RFLP, were discordant. We report reanalysis of our data using the more accurate TaqMan assay.

\section{RESULTS}

\section{Comparison of genotype results obtained by TaqMan assay vs. those obtained by RFLP}

Each of the available 167 specimens was regenotyped using TaqMan methodology, for each of the genotypes (ESR1 XbaI and PvuII; ESR2-01 and -02) reported by Ntukidem et al. ${ }^{2}$ Each TaqMan genotype assay was performed on two separate occasions. All replicate TaqMan assay results for each genotype were identical.

Concordance between the results of TaqMan and RFLP genotyping assays for ESR1 PvuII, ESR2-01, and -02 was $\geq 98 \%$. TaqMan genotyping was successfully determined for ESR $1 \mathrm{XbaI}$ in 157 of the 167 available DNA specimens (see Supplementary Figure $\mathbf{S 1}$ online). Of these, 17 results (10.8\%) showed discordance on comparison.

\section{Correlation between genotype and phenotype}

Associations between ESR1 Pvull, ESR2, and CYP2D6 genotypes, and lipid levels and clinical features. In the findings from the reanalysis, we observed that the previously reported associations between menopausal status and serum lipid concentrations at baseline, and between tamoxifen use and tamoxifen-associated change in lipid levels, remained unchanged. Likewise, as in the original report, changes in triglyceride levels were significantly associated with ESR2-02 genotypes, whereas there was no association between either the ESR1 PvuII or the CYP2D6 genotype and changes in serum lipid concentrations after tamoxifen treatment (see Supplementary Table S1 online and Supplementary Figure S1 online).

Associations between ESR1 Xbal and lipids. We previously reported ${ }^{2}$ a statistically significant association between total cholesterol level at baseline before tamoxifen treatment in all women and, in subgroup analysis, in premenopausal women with ESR1 $\mathrm{Xbal}$ genotypes. In the reanalysis with TaqMan assay genotyping, these associations were not observed $(P=0.23$ and 0.94 , respectively; Table 1). Likewise, previously reported associations were no longer detected between LDL-cholesterol level in the entire data set and in the premenopausal subgroup with ESR1 XbaI genotypes ( $P=0.15$ and 0.93 , respectively; Table 1).

In agreement with the findings using RFLP genotyping, after 4 months of treatment with tamoxifen, there were no detectable associations between TaqMan-analyzed ESR1 XbaI genotypes and levels of triglycerides, HDL cholesterol, and LDL cholesterol (Table 2). Although the previously reported association was again observed between the GG genotype of XbaI and greater reduction in total cholesterol level, it was not statistically significant in the reanalysis (reduction in total cholesterol for each genotype: AA $(n=31)-28 \mathrm{mg} / \mathrm{dl} ; \mathrm{AG}(n=30)-13 \mathrm{mg} / \mathrm{dl}$; and GG $(n=13)-41 \mathrm{mg} / \mathrm{dl} ; P=0.06$ ) (Table 2$)$. Also, the previously reported correlations between XbaI genotypes and triglyceride levels in premenopausal women were not observed in the reanalysis (Figure 1b). Although the changes in HDL-cholesterol levels associated with the XbaI genotypes were quantitatively similar to those previously observed in this group of patients, these changes failed to reach statistical significance in the reanalysis $(P=0.09)$ (Figure 1c). As before, no statistically significant associations were detected between XbaI genotypes and

Table 1 Mean baseline lipid concentrations according to ER- $a$ Xbal genotype and menopausal status

\begin{tabular}{|c|c|c|c|c|c|}
\hline ER-a Xbal genotype & Lipid particle & $\mathrm{AA}(n=48)$ & AG $(n=58)$ & $\mathrm{GG}(n=16)$ & $P$ value ${ }^{a}$ \\
\hline \multirow[t]{3}{*}{ All women $(N=122)^{b}$} & Total cholesterol & $227(213,241)^{c}$ & $215(204,225)$ & $234(212,256)$ & 0.23 \\
\hline & Triglycerides & $141(120,161)$ & $135(106,164)$ & $131(101,160)$ & 0.69 \\
\hline & LDL cholesterol & $137(125,149)$ & $124(115,133)$ & $144(126,162)$ & 0.15 \\
\hline \multirow[t]{4}{*}{ Premenopausal $(n=48)$} & & $(N=17)$ & $(N=28)$ & $(N=3)$ & \\
\hline & Triglycerides & $141(102,180)$ & $115(67,163)$ & $92(23,161)$ & 0.19 \\
\hline & HDL cholesterol & $59(54,65)$ & $70(63,76)$ & $68(6,130)$ & 0.89 \\
\hline & LDL cholesterol & $123(100,146)$ & $118(106,130)$ & $122(41,202)$ & 0.93 \\
\hline \multirow[t]{2}{*}{ Postmenopausal $(n=74)$} & & $(N=31)$ & $(N=30)$ & $(N=13)$ & \\
\hline & Total cholesterol & $235(218,251)$ & $220(206,233)$ & $240(217,263)$ & 0.30 \\
\hline
\end{tabular}

$\mathrm{HDL}$, high-density lipoprotein; LDL, low-density lipoprotein.

${ }^{a} P$ values are calculated for the differences between the AA/AG and GG alleles. ${ }^{b}$ Number of ER-a genotypes available. ${ }^{\mathrm{C}} \mathrm{Mean}$ baseline lipid particle concentration (95\% $\mathrm{Cl}$ ). 
Table 2 Association between change in total cholesterol concentration at 4 months and ER-a Xbal genotypes in the postmenopausal group

\begin{tabular}{lcccc}
\hline & & ER-a Xbal genotype & & \\
\cline { 2 - 4 } Lipid particle & AA $(\boldsymbol{n}=31)$ & AG $(\boldsymbol{n}=30)$ & GG $(\boldsymbol{n}=13)$ & \\
\hline Total cholesterol & $-28(-40,-16)^{\mathrm{b}}$ & $-13(-22,-4)$ & $-41(-62,-20)$ & 0.06 \\
\hline Triglycerides & $28(4.5,51)$ & $29(-33,90)$ & $27(-37,92)$ & 0.98 \\
\hline HDL cholesterol & $-1(-5.8,3.6)$ & $1.9(-1.0,4.8)$ & $-1.5(-9.2,6.1)$ & 0.61 \\
\hline LDL cholesterol & $-32(-43,-21)$ & $-16(-25,-8)$ & $-39(-60,-18)$ & \\
\hline
\end{tabular}

$\mathrm{HDL}$, high-density lipoprotein; LDL, low-density lipoprotein.

ap values are calculated for the differences between baseline and 4 months concentration for the AA/AG vs. the GG alleles. ${ }^{b}$ Change in serum lipid particle concentration is shown in $\mathrm{mg} / \mathrm{dl}$ with $95 \% \mathrm{Cl}$.
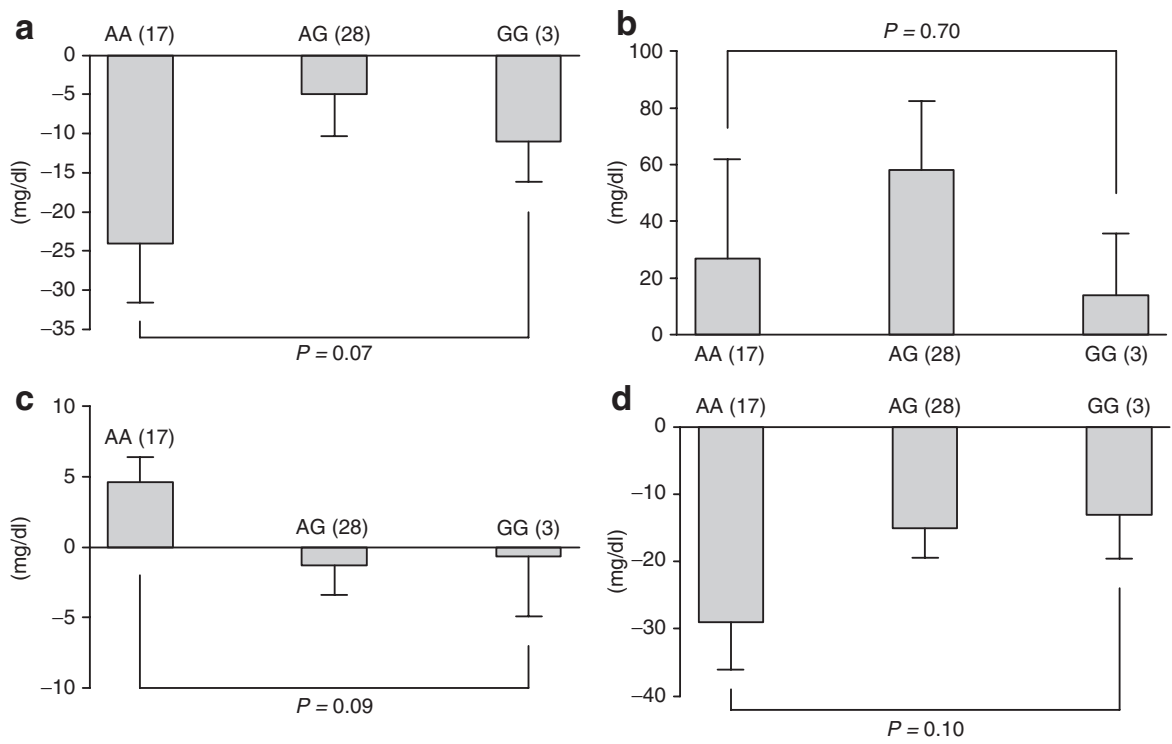

Figure 1 Response of serum lipid particle levels to tamoxifen in premenopausal women according to their ESR1 Xbal genotypes: (a) total cholesterol, (b) triglycerides, (c) high-density lipoprotein cholesterol, and (d) low-density lipoprotein cholesterol. The bars show the mean change in serum lipid concentration in $\mathrm{mg} / \mathrm{dl}$, and the error bars are the standard error of the means. The $y$ axis indicates the direction of the change in lipid concentration. Shown in parentheses next to each genotype is the number of subjects in each genotype group. $P$ value shown is for gene-dose effect.

tamoxifen-induced changes in total cholesterol levels $(P=0.07)$ (Figure 1a) and LDL-cholesterol levels $(P=0.10)$ (Figure 1d).

\section{DISCUSSION}

Tamoxifen exerts its biological action through binding with its receptors, ER $\alpha$ and $\beta$, encoded by the genes ESR 1 and 2, respectively. Using an older assay, we had reported selected associations between SNPs in these genes and the favorable effect of tamoxifen on lipid profiles. In this report, we have identified that, for one of these alleles (ESR1 XbaI), reanalysis with the more accurate assay, TaqMan, indicated that $\sim 10 \%$ of the previously assigned genotypes were incorrect. In contrast, our previously reported genotypes for ESR1 PvuII, ESR2, and CYP2D6 were correct (98-100\% concordance between assays).

In a reanalysis of the clinical associations of inherited germline SNPs, using the genotypes derived from the TaqMan assays, we no longer detected the previously reported statistically significant association between ESR1 Xba1 genotype and baseline triglyceride level in all women or between ESR1 Xba1 genotype and baseline triglyceride or LDL-cholesterol level in the premenopausal subset. Likewise, in postmenopausal women, we no longer detected any statistically significant associations between ESR1 XbaI genotype and tamoxifen-induced changes in total cholesterol level, nor in premenopausal women between ESR1 XbaI genotype and tamoxifen-induced changes in triglyceride or HDL-cholesterol level. Most of these differences in the findings are attributable to genotype reassignment in the $\sim 10 \%$ of subjects in whom the genotype had initially been incorrectly assigned on the basis of RFLP genotyping.

These data may have relevance to many other studies using RFLP assays in general but specifically to those using it for detecting variants in ESR1, originally described by Herrington et al. ${ }^{6}$ We are not certain of the cause of the original genotyping errors. We believe that incomplete digestion of the PCR product by the XbaI enzyme may have contributed to the error, possibly because of a reagent problem, a tube heating block problem, or an unknown factor in the DNA sample that partially blocked the enzyme. Alternatively, there may have been some peak suppression on the instrument that was used to analyze the digested PCR products. This circumstance could result from too little or too much DNA in the sample, a manufacturing problem with the chip, or an imperfection in the corresponding reagent. We 
repeated the RFLP assay on the DNA samples from these subjects and obtained the correct genotyping results, thereby indicating that there is no problem specific to the SNP that affects the assay. Given that the frequencies of occurrence of these errors are small, they were not observed in our original development and validation of the RFLP assay. In summary, we believe that these are random errors that may or may not be present in assays conducted by other investigators.

It is possible that some of the differences in our observations, such as the tamoxifen-induced changes in total cholesterol levels in postmenopausal women $(P=0.06$; Table 2$)$ and in HDL-cholesterol levels in premenopausal women $(P=0.09$, Figure 1c), are attributable to loss of statistical power because not all the specimens were available for the reanalysis. However, power calculations for the results presented in Tables 1 and 2 (current version), and their corresponding tables (Table 3a,b in the original version), do not support this hypothesis. It is clear that if the number of samples assayed were decreased, but the size of the genetic effects were assumed to be the same, then the $5 \%$ reduction in sample size would have led to only a $3-4 \%$ reduction in power. Therefore, the difference between the original report and the reanalysis is unlikely to be due to the decrease in the number of samples per se. Instead, it appears that the loss of statistical power is the result of a decrease in the magnitude of the genetic effects observed, which in turn is a consequence of the reassignment of genotypes made necessary by the corrections in the genotyping results. The following other observations from the original submission have not changed: (i) circulating lipid levels are decreased in women taking tamoxifen, a finding that is consistent with other reports; (ii) changes in triglyceride levels were statistically associated with ESR2-02 genotype; and (iii) there was no association between either ESR1 PvuII or CYP2D6 genotype and changes in serum lipid concentrations after treatment with tamoxifen.

\section{METHODS}

Subjects, study design, sample collection, and lipid analysis. The subjects, study design, sample collection, and lipid analysis details have been previously described, including exclusion of perimenopausal women and patients taking lipid-lowering medications (ClinicalTrials. gov identifier NCT00228930) (ref. 2). Archived genomic DNA specimens were selected for reanalysis using the TaqMan assay. Of the 176 specimens included in the original report by Ntukidem et al., 9 had been exhausted by previous testing, and therefore 167 (95\%) of the original specimens were available for this reanalysis (see Supplementary Figure S1 online).

Genotyping. In our previous report, ${ }^{2}$ genotyping for ESR 1 was conducted using the RFLP method, previously described in studies of the effects of estrogens on lipid levels in postmenopausal women, ${ }^{6}$ whereas ESR2 genotyping was performed using the TaqMan method. ${ }^{2}$ In this study, to ensure uniform quality control, all specimens were regenotyped for ESR1 and ESR2 using TaqMan assays in accordance with the manufacturer's instructions (Applied Biosystems, Foster City, CA) as previously described and validated ${ }^{7}$ (http://snp500cancer.nci.nih.gov; ESR2-01: rs no. 1256049; ESR2-02: rs no. 4986938). ESR1 genotypes for rs2234693 (ESR1 PvuII) and rs9340799 (ESR1 XbaI) were determined using the following TaqMan assays: C_3163590_10 for the rs no. 2234693 and C_3163591_10 for the rs no. 9340799. In a previous publication by Consortium on Breast Cancer Pharmacogenomics (COBRA) investigators, the concordance rate between these two assays and manual DNA sequencing was $>99 \%$ in 220 subjects. ${ }^{7}$ As a further confirmation, each specimen was genotyped twice using TaqMan assays, and any results that were discordant with the previous RFLP assay results were retested by DNA sequencing. All samples were also regenotyped for ESR2 using the TaqMan assay previously described. ${ }^{2}$ In our previous report, the results of the CYP2D6 genotyping were from at least two different methodologies that showed $100 \%$ concordance, and therefore we did not repeat CYP2D6 genotyping in this genotyping analysis.

Statistical analysis. Detailed methods for statistical analytical correlations among clinical factors, lipids, and genotypes have been previously described, ${ }^{2}$ and the reanalyses of these correlations in the current report were performed identically. In addition, a similar reanalysis was performed by a completely separate and independent statistical team with identical results (see Supplementary Table S1 online, Supplementary Figure S1 online, and Acknowledgments).

SUPPLEMENTARY MATERIAL is linked to the online version of the paper at http://www.nature.com/cpt

\section{ACKNOWLEDGMENTS}

This study was supported in part by Pharmacogenetics Research Network grant U-01 GM61373 (D.A.F.), which supports the Consortium on Breast Cancer Pharmacogenomics (COBRA); the Damon Runyon-Lilly Clinical Investigator Award Cl-3 from the Damon Runyon Cancer Research Foundation (V.S.); Indiana University GCRC grant M01RR00750 from the $\mathrm{NIH}$; University of Michigan GCRC grant M01-RR00042 from the $\mathrm{NIH}$; and the Fashion Footwear Charitable Foundation of New York/QVC Presents Shoes on Sale (D.F.H.). We thank Marylyn Ritchie, Associate Professor, Vanderbilt University Center for Human Genetics Research, for her thorough reanalysis of the data in this report (see Supplementary Table S1 online and Supplementary Figure $\mathbf{S} 1$ online). A description of the study design can be found at http://www.ClinicalTrials.gov (NCT00228930).

\section{CONFLICT OF INTEREST}

D.F.H. has received research funding from AstraZeneca, and the Consortium on Breast Cancer Pharmacogenomics has received research funding from Pfizer and Novartis. AstraZeneca markets tamoxifen (although off patent), and Pfizer and Novartis make aromatase inhibitors, which are indirectly addressed in this paper. None of the authors reports any conflicts of interest regarding these results.

๑) 2010 American Society for Clinical Pharmacology and Therapeutics

1. MacGregor, J.I. \& Jordan, V.C. Basic guide to the mechanisms of antiestrogen action. Pharmacol. Rev. 50, 151-196 (1998).

2. Ntukidem, N.I. et al.; Consortium on Breast Cancer Pharmacogenomics. Estrogen receptor genotypes, menopausal status, and the lipid effects of tamoxifen. Clin. Pharmacol. Ther. 83, 702-710 (2008).

3. Massart, F. Human races and pharmacogenomics of effective bone treatments. Gynecol. Endocrinol. 20, 36-44 (2005).

4. Stearns, V. et al. Active tamoxifen metabolite plasma concentrations after coadministration of tamoxifen and the selective serotonin reuptake inhibitor paroxetine. J. Natl. Cancer Inst. 95, 1758-1764 (2003).

5. Jin, Y.et al. CYP2D6 genotype, antidepressant use, and tamoxifen metabolism during adjuvant breast cancer treatment. J. Natl. Cancer Inst. 97, 30-39 (2005).

6. Herrington, D.M. et al. Estrogen-receptor polymorphisms and effects of estrogen replacement on high-density lipoprotein cholesterol in women with coronary disease. N. Engl. J. Med. 346, 967-974 (2002).

7. Onitilo, A.A. et al. Estrogen receptor genotype is associated with risk of venous thromboembolism during tamoxifen therapy. Breast Cancer Res. Treat. 115, 643-650 (2009). 